\title{
The choice of operation timing of laparoscopic cholecystectomy (LC) after percutaneous transhepatic gallbladder drainage (PTGBD) for acute cholecystitis: a retrospective clinical analysis
}

\author{
Jianheng Bao $^{1 \#}$, Jianxiong Wang ${ }^{2 \#}$, Haitao Shang ${ }^{1}$, Chengfei Hao ${ }^{1}$, Junjian Liu ${ }^{1}$, Delin Zhang ${ }^{1}$, Shuwang Han $^{1}$, \\ Zhonglian $\mathrm{Li}^{1}$ \\ ${ }^{1}$ Department of Hepatobiliary and Pancreatic Surgery, Tianjin Nankai Hospital, Tianjin, China; ${ }^{2}$ Department of Ultrasonography, Tianjin Nankai \\ Hospital, Tianjin, China \\ Contributions: (I) Conception and design: J Bao; (II) Administrative support: Z Li; (III) Provision of study materials or patients: J Wang; (IV) \\ Collection and assembly of data: J Bao; (V) Data analysis and interpretation: J Bao; (VI) Manuscript writing: All authors; (VII) Final approval of \\ manuscript: All authors. \\ "These authors contributed equally to this work. \\ Correspondence to: Zhonglian Li. Department of Hepatobiliary and Pancreatic Surgery, Tianjin Nankai Hospital, No. 6 ChangJiang Road, Nankai \\ District, Tianjin 300100, China. Email: Nkyylz1@163.com.
}

Background: This study aimed to evaluate the timing of laparoscopic cholecystectomy (LC) after percutaneous transhepatic gallbladder drainage (PTGBD).

Methods: Patients with acute moderate to severe cholecystitis treated by LC after PTGBD in the Department of Hepatobiliary and Pancreatic Surgery, Nankai Hospital (N-362) between January 2017 and August 2019were retrospectively enrolled into this study. According to the interval times from PTGBD to LC, the patients were divided into six groups, including group A (105 cases, within 1 week), group B (62 cases, 1-2 weeks), group C (34 cases, 3-4 weeks), group D (54 cases, 5-8 weeks), group E (24 cases, 9-12 weeks), and group F (83 cases, over 12 weeks). The gender, age, hospital stay, duration of operation, rate of conversion to laparotomy, incidence of complications, and hospitalization expenses of the six groups were evaluated and compared.

Results: Of the 362 cases of LC, 346 patients were operated successfully (95.6\%), 10 were converted to laparotomy (2.8\%), 16 had various complications (4.4\%), and 2 died $(0.6 \%)$. There were no significant differences between groups in the gender ratio, complication rate, and rate of conversion to laparotomy. The hospital stay and hospitalization expenses in group A were the least and significantly lower than those in other groups $(\mathrm{P}<0.01)$, and the duration of operation in group $\mathrm{D}$ was the longest and significantly higher than that in groups $\mathrm{A}, \mathrm{B}, \mathrm{E}$, and $\mathrm{F}(\mathrm{P}<0.05)$.

Conclusions: For non-elderly patients diagnosed with acute moderate to severe cholecystitis with an anesthesia risk score [American Society of Anesthesiologists (ASA)] $\leq 2, \mathrm{LC}$ is recommended to be performed within 1 week after PTGBD surgery. If delayed LC is performed within 2 to 8 weeks after PTGBD, the operation time will be longer due to inflammatory edema and fibrous adhesion of the gallbladder triangle. If PTGBD is performed for more than 2 months and the clinical circumstances are good, delayed LC can be considered to reduce the inconvenience of patients with a long-term catheter as much as possible.

Keywords: Percutaneous transhepatic gallbladder drainage (PTGBD); laparoscopic cholecystectomy (LC); acute cholecystitis

Submitted Jun 23, 2021. Accepted for publication Aug 05, 2021.

doi: 10.21037/apm-21-1906

View this article at: https://dx.doi.org/10.21037/apm-21-1906 


\section{Introduction}

Acute cholecystitis is a common cause of acute abdomen often accompanied by cholelithiasis. Continuous exploration and improvement have resulted in the diagnosis and treatment of acute cholecystitis becoming increasingly standardized, with a high cure rate and low mortality rate. The Tokyo Guidelines 2018 (TG18) are an important reference guide and have formulated the diagnostic criteria, severity classification, drainage mode selection, and operation related precautions of acute cholecystitis (1-3).

Laparoscopic cholecystectomy (LC) is currently considered as the main radical treatment for acute cholecystitis and is classified as early LC (within 72 hours or 1 week of onset) and delayed LC (after antibiotics are given to control inflammation) (4). In some cases of acute cholecystitis, complications and mortality are significantly increased due to systemic or local conditions. In these patients, percutaneous transhepatic gallbladder drainage (PTGBD) to reduce local gallbladder edema, control infection, and improve systemic symptoms to create better surgical conditions for subsequent surgical treatment should be performed $(5,6)$. Therefore, some studies have focused on the PTGBD and LC. Chun and co-workers clarified the surgical outcomes after PTGBD + LC for the management of patients with moderate to severe acute cholecystitis (7). $\mathrm{Yu}$ and co-workers presented the primary experience on a new approach of cholangiography via PTGBD for preoperative delineation of biliary anatomy (8). However, there is no consensus on when to perform LC after PTGBD $(3,6)$.

This study aimed to explore the timing of LC after PTGBD, by investigating and comparing gender, age, hospital stay, duration of operation, rate of conversion to laparotomy, the incidence of complications, and hospitalization expenses of patients with different interval times from PTGBD to LC.

We present the following article in accordance with the STROBE reporting checklist (available at https://dx.doi. org/10.21037/apm-21-1906).

\section{Methods}

\section{Patients}

A retrospective study was conducted on 474 patients with acute cholecystitis, including 264 males and 210 females aged 18-92 years old, with an average age of 65.32 years old. All patients presented between January 2017 and August
2019. Underlying conditions included acute suppurative cholecystitis, gallbladder expansion, gallbladder wall thickening, pericholecystic fluid collection, and gallbladder perforation, and all patients underwent PTGBD guided by ultrasound within 1-2 days following admission. Seven patients with chills and three with gallbladder hemorrhage recovered and stopped bleeding after treatment, respectively, while the drainage tube fell off in five, and no serious complications occurred after the second puncture. LC was performed in 362 patients after PTGBD, of which 167 who only underwent PTGBD for the first hospital admission were hospitalized twice. Another 112 cases underwent other operations after PTGBD. Among the 362 patients, 39 underwent endoscopic retrograde cholangiopancreatography (ERCP) and endoscopic sphincterotomy (EST) 1-2 days before LC. According to the interval times from PTGBD to $\mathrm{LC}$, patients were divided into six groups, including group A (105 cases, within 1 week), group B (62 cases, 1-2 weeks), group C (34 cases, 3-4 weeks), group D (54 cases, 5-8 weeks), group E (24 cases, 9-12 weeks), group F (83 cases, over 12 weeks) (Figure 1 ).

All procedures performed in this study involving human participants were in accordance with the Declaration of Helsinki (as revised in 2013). This study was reviewed and approved by the Ethics Committee of Tianjin Nankai Hospital (No. NKYY_YXKT_IRB_2019_004_01). Written informed consent was obtained from all study participants.

\section{Inclusion criteria}

The inclusion criteria for acute cholecystitis consist of local, systemic, and imaging evidence, including the following (3): (I) local manifestations: Murphy's sign and right upper abdominal pain, tenderness, or mass; (II) systemic manifestations: fever, raised C-reactive protein and white blood cell (WBC); (III) imaging finding evidence of acute cholecystitis. Acute cholecystitis was classified into three levels according to TG18 (9).

\section{Exclusion criteria}

The exclusion criteria were as follows: (I) patients with grade I and II acute cholecystitis, whose Charlson Comorbidity Index (CCI) $\geq 6$ and American Society of Anesthesiologists Physical Status Classification (ASA-PS) $\geq 3$; (II) patients with grade III acute cholecystitis complicated with nervous and respiratory system insufficiency or with jaundice (total bilirubin $\geq 35.4 \mu \mathrm{mol} / \mathrm{L}$ ); (III) CCI $\geq 4$ and ASA-PS $\geq 3$ (9). 


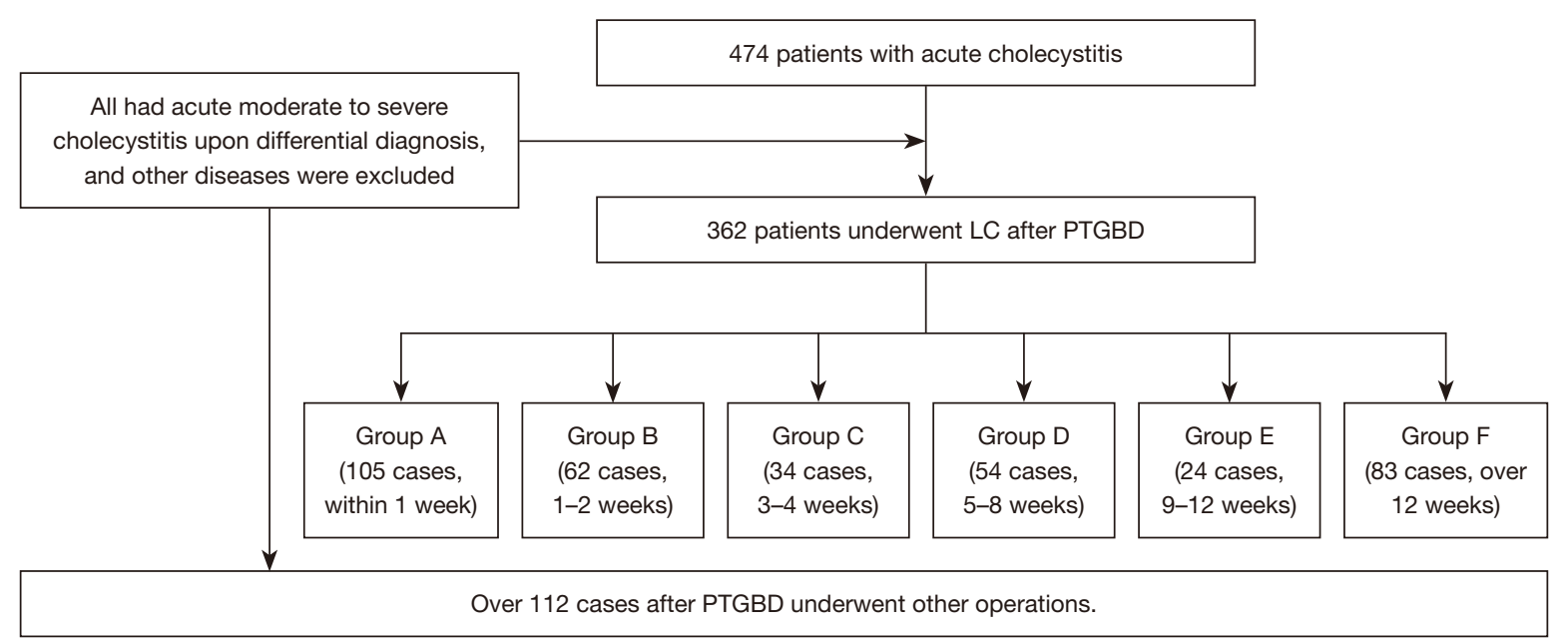

Figure 1 Flow diagram of the included and excluded patients. LC, laparoscopic cholecystectomy; PTGBD, percutaneous transhepatic gallbladder drainage.

\section{Procedure of PTGBD}

Patients with contradictions for puncture were excluded before performing PTGBD. Subsequently, supportive treatment and antibiotics were given according to the condition of the patient. During the procedure, patients were usually placed in a supine position and anesthetized with $2 \%$ lidocaine after ultrasound positioning from the right intercostal space. Using a percutaneous transhepatic approach, an 8 or $10 \mathrm{Fr}$ disposable puncture trocare was inserted into the gallbladder to place a pig-tail tube as close as possible to the neck of the gallbladder. Bile was then extracted for decompression and sent to bacterial culture. The front end of the drainage tube was confirmed to be coiled in the gallbladder, and the drainage tube was fixed on the right abdominal wall. After the operation, it was necessary to redress internal milieu disorder and commence antibiotics (10).

\section{LC procedure}

All patients were given intravenous and volatile mixed anesthesia and tracheal intubation, followed by a three-hole or four-hole method of LC. The incision was made under or above the umbilicus to place the laparoscope under a $\mathrm{CO}_{2}$ environment with aerated pressure of $10-12 \mathrm{mmHg}$. The region was first generally explored, then the gallbladder tube and anterior and posterior branches of the gallbladder artery were closed with an absorbable non-metallic clip. The gallbladder bed was then separated by electrocautery and the gallbladder was completely removed, with a drainage tube placed in the right liver or foramen of Winslow in all patients (11). After successful puncture, the drain was secured to the right abdominal wall using a catheter holder and a waterproof patch was applied (Figure 2).

\section{Retrospective analysis}

The retrospective analysis was conducted to compare the gender, age, hospital stay, duration of operation, rate of conversion to laparotomy, the incidence of complications, and hospitalization expenses of patients with different interval times from PTGBD to LC.

\section{Statistical analysis}

All data were analyzed using SPSS 20.0 software. Categorical data were expressed as $\mathrm{n}(\%)$, and the differences among groups were examined by chi-square analysis. $\mathrm{P}<0.05$ was considered statistically significant. The measurement data were expressed as mean \pm standard deviation $(\mathrm{SD})$, and the comparisons were examined by one-way analysis of variance (ANOVA) between groups, $\alpha=0.05$.

\section{Results}

\section{Summary of gender ratio, complications rate, and rate of conversion to laparotomy}

Of the 362 cases, LC was successfully performed in 346, with a success rate of $95.6 \%$. Further, the gender ratio, 

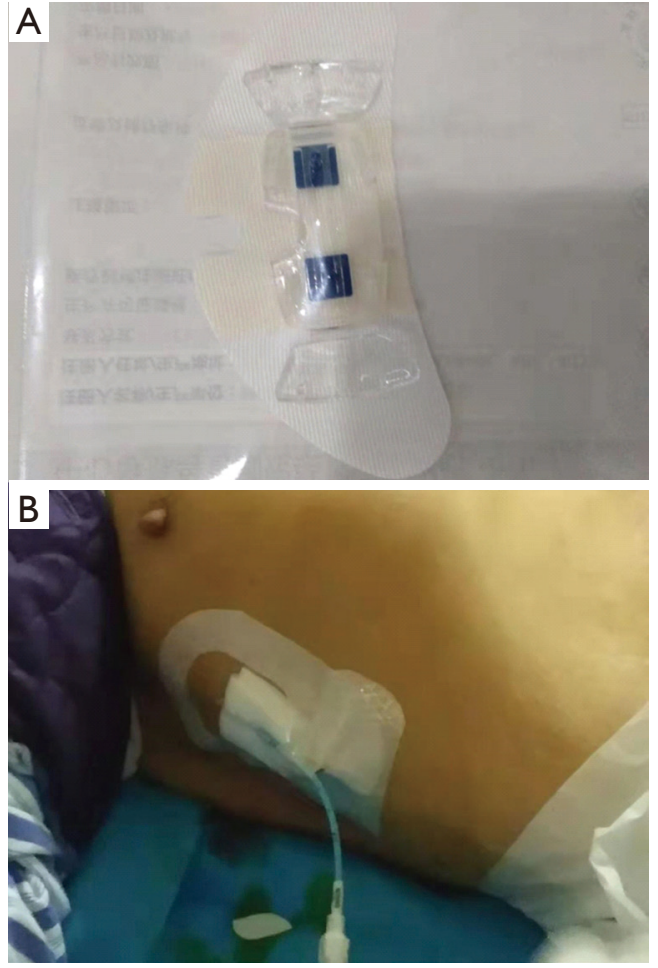

Figure 2 Fixation method of the PTGBD drain. (A) is the drain fixation device. (B) is a typical drawing of a PTGBD drain fixed to a patient. PTGBD, percutaneous transhepatic gallbladder drainage.

complications rate, and rate of conversion to laparotomy among patients with different interval time from PTGBD to LC were compared and analyzed, as shown in Table 1. Results showed that there were no significant differences among different groups. In addition, 10 cases $(2.8 \%)$ were transferred to laparotomy, among whom three patients had gallstones in the neck of the gallbladder which compressed the triangle of the gallbladder and led to an inability to identify the relationship among the common hepatic duct, cystic duct, and cystic artery. Five patients were diagnosed with type I and II Mirizzi syndrome during the operation, and laparotomy was required to explore the common hepatic duct and common bile duct. One patient developed a cholecystoduodenal fistula due to inflammation around the gallbladder and underwent duodenal repair, while in another, a gallbladder colon fistula was found, and the patient underwent colon repair.

Complications occurred in 16 patients (4.4\%) including two during anesthesia, of whom one died. Postoperative myocardial infarction occurred in two patients, one of whom also died, while in three patients, the lateral wall of the hepatic duct was damaged during the operation and was repaired by intraoperative absorbable suture without sequela. Postoperative biliary fistula occurred in two patients, which was treated by abdominal puncture and drainage, and residual calculi of the common bile duct were found in four patients after operation, which were cured by duodenal endoscopic stone extraction. Gallbladder cancer was found in three patients, who then underwent a second operation to remove the $\mathrm{IV}$ and $\mathrm{V}$ segments of the liver and lymph node dissection. The overall mortality rate was $0.6 \%$.

\section{Summary of age, duration of operation, hospital stay, and hospitalization expenses}

The age, duration of operation, hospital stay, and hospitalization expenses among patients with different interval times from PTGBD to LC were compared and are shown in Table 2. Results showed that there were significant differences among different groups. The duration of operation was $16-261 \mathrm{~min}$, with an average of $76.57 \pm 41.54 \mathrm{~min}$, and was shortest when LC was performed within 1 week after PTGBD. However, the average operation time was the longest when LC was performed within 5-8 weeks after PTGBD. The hospital stay was 4-106 days, with an average of $17.08 \pm 9.91$ days, and was shortest and longest for group $\mathrm{A}$ and $\mathrm{F}$, respectively. The hospitalization expenses were $46,551.18 \pm 19,872.19$ yuan, with the minimum and maximum average cost for group A and group F, respectively.

As there were significant differences in the age, duration of operation, hospital stay, and hospitalization expenses among the six groups, they were further compared in pairs, as shown in Table 3. Statistical differences were found between group $\mathrm{A}$ and group $\mathrm{F}(\mathrm{P}<0.05)$ in patient age, indicating early LC was mainly performed in non-elderly patients ( $<75$ years). There were also statistical differences in age distribution between group $\mathrm{D}$ and groups $\mathrm{B}, \mathrm{E}$, and $\mathrm{F}$ $(\mathrm{P}<0.05)$. There was a statistical difference in operation time between group $\mathrm{D}$ and groups $\mathrm{A}, \mathrm{B}, \mathrm{E}$, and $\mathrm{F}(\mathrm{P}<0.05)$, but not between group $\mathrm{D}$ and group $\mathrm{C}(\mathrm{P}>0.05)$ which indicates inflammation and edema did not completely disappear within 2-8 weeks after PTGBD, and fibrous adhesions were more severe, leading to difficulty performing LC. Compared with other groups, the hospital stay and hospitalization expenses in group A were significantly lower $(\mathrm{P}<0.01)$, while there were no significant differences in the incidence of complications and the rate of conversion to laparotomy. The hospital stay and hospitalization expenses 
Table 1 Comparison of gender, complications, and conversion to laparotomy among patients with different interval times from PTGBD to LC

\begin{tabular}{llcccc}
\hline Group & Interval time from PTGBD to LC & Number of cases & Gender (male/female) & Complications (no/yes) & Laparotomy (no/yes) \\
\hline A & Within 1 week & 105 & $58 / 47$ & $103 / 2$ & $104 / 1$ \\
B & 1-2 weeks & 62 & $37 / 25$ & $58 / 4$ & $33 / 1$ \\
C & 3-4 weeks & 34 & $21 / 13$ & $54 / 0$ & $34 / 0$ \\
D & 5-8 weeks & 54 & $33 / 21$ & $23 / 1$ & $50 / 4$ \\
E & 9-12 weeks & 24 & $11 / 13$ & $75 / 8$ & $24 / 0$ \\
F & Over 12 weeks & 83 & $44 / 39$ & 9.267 & $50 / 3$ \\
$\chi^{2}$ & & & 2.690 & 0.059 \\
P & & & 0.748 & 0.256 \\
\hline
\end{tabular}

PTGBD, percutaneous transhepatic gallbladder drainage; LC, laparoscopic cholecystectomy.

Table 2 Comparison of age, duration of operation, hospital stay and hospitalization expenses among patients with different interval times from PTGBD to LC

\begin{tabular}{|c|c|c|c|c|c|c|}
\hline Group & $\begin{array}{l}\text { Interval time from } \\
\text { PTGBD to LC }\end{array}$ & $\begin{array}{l}\text { Number of } \\
\text { cases }\end{array}$ & $\begin{array}{l}\text { Age } \\
\text { (year) }\end{array}$ & $\begin{array}{l}\text { Duration of operation } \\
\text { (min) }\end{array}$ & Hospital stay (day) & $\begin{array}{l}\text { Hospitalization expense } \\
\text { (yuan) }\end{array}$ \\
\hline A & Within 1 week & 105 & $63.18 \pm 11.14$ & $70.58 \pm 40.31$ & $10.98 \pm 5.73$ & $36,038 \pm 13,108.35$ \\
\hline C & 3-4 weeks & 34 & $63.82 \pm 14.69$ & $80.32 \pm 41.18$ & $21.47 \pm 8.95$ & $53,455 \pm 22,411.02$ \\
\hline $\mathrm{F}$ & Over 12 weeks & 83 & $66.93 \pm 11.65$ & $72.64 \pm 36.51$ & $22.41 \pm 13.58$ & $57,606 \pm 25,734.95$ \\
\hline$t$ value & & & 2.239 & 2.465 & 18.596 & 14.745 \\
\hline$P$ value & & & 0.050 & 0.033 & $<0.001$ & $<0.001$ \\
\hline
\end{tabular}

PTGBD, percutaneous transhepatic gallbladder drainage; LC, laparoscopic cholecystectomy.

in group B were significantly fewer than in groups $\mathrm{C}, \mathrm{E}$, and $\mathrm{F}$, and groups $\mathrm{C}$ and $\mathrm{F}(\mathrm{P}<0.05)$, respectively, and the hospital stay and hospitalization expenses in group $\mathrm{D}$ were also significantly lower than those in groups $\mathrm{C}$ and $\mathrm{F}$ $(\mathrm{P}<0.05)$.

\section{Discussion}

Pain and local infection in acute cholecystitis, especially suppurative cholecystitis, are severe. If the diagnosis is clear and surgical contraindications are excluded, active LC is an effective treatment method, although LC has the potential to cause complications of artificial pneumoperitoneum, complications of catheter puncture, surgical injuries, complications of puncture holes in the abdominal wall, and so on (12). TG18 showed there were no significant differences in operative time, complication rate, length of hospital stay, and hospitalization costs between early LC and delayed LC. However, surgical treatment within 24 hours of onset was not superior to that within 72 hours of onset. While it is advocated that patient assessment should be optimized and surgical treatment should be carried out as early as possible, emergency surgery is not required (2).

With advanced age and poor systemic function, local infection of acute moderate to severe cholecystitis is prone to immune dysfunction, disseminated intravascular coagulation (DIC), and septic shock (13). Radder first reported that $\mathrm{PTGBD}$ could reduce gallbladder pressure and drain infected bile under the guidance of ultrasound. 
Table 3 Comparison of age, duration of operation, hospital stay, and hospitalization expenses between two groups

\begin{tabular}{|c|c|c|c|c|}
\hline Groups & Age (P value) & Duration of operation (P value) & Hospital stay ( $P$ value) & Hospitalization expense ( $P$ value) \\
\hline A vs. C & 0.785 & 0.231 & $<0.001^{\star \star \star}$ & $<0.001^{\star \star \star}$ \\
\hline A vs. D & 0.184 & $0.001^{\star \star \star}$ & $<0.001^{\star \star \star}$ & $0.003^{\star \star}$ \\
\hline A vs. E & 0.251 & 0.861 & $<0.001^{\star \star \star}$ & $<0.001^{\star \star \star}$ \\
\hline B vs. C & 0.668 & 0.708 & $0.004^{\star \star}$ & $0.028^{\star}$ \\
\hline B vs. D & $0.049^{*}$ & $0.036^{\star}$ & 0.381 & 0.958 \\
\hline B vs. E & 0.633 & 0.626 & $0.024^{*}$ & 0.091 \\
\hline B vs. F & 0.318 & 0.527 & $<0.001^{\star \star \star}$ & $<0.001^{\star \star \star}$ \\
\hline C vs. F & 0.203 & 0.361 & 0.604 & 0.264 \\
\hline D vs. E & $0.050^{*}$ & $0.038^{\star}$ & 0.122 & 0.106 \\
\hline D vs. F & $0.002^{\star \star}$ & $0.005^{\star \star}$ & $0.002^{\star \star}$ & $<0.001^{\star \star \star}$ \\
\hline Evs. F & 0.819 & 0.964 & 0.457 & 0.208 \\
\hline
\end{tabular}

${ }^{\star}, \mathrm{P} \leq 0.05 ;{ }^{\star \star}, \mathrm{P} \leq 0.01 ;{ }^{\star \star \star}, \mathrm{P} \leq 0.001$.

This method only requires local anesthesia and bedside ultrasound positioning, with low requirements for equipment materials, and is suitable for most patients, especially the elderly and long-term bedridden patients $(14,15)$. Although PTGBD has an obvious effect in the early treatment of acute cholecystitis and can be used as an alternative to LC treatment, the long-term indwelling of the puncture tube can produce more adverse reactions, such as the recurrence of acute cholecystitis, acute cholangitis, and acute pancreatitis. Hung et al. retrospectively analyzed 625 patients with acute cholecystitis who underwent PTGBD and found that while the complication rate was not high in the early stage after treatment (16), with prolonged indwelling time of the drainage tube, the cumulative incidence of biliary adverse reactions increased up to $29.8 \%$, with a median occurrence time of 4.27 months after PTGBD.

Therefore, for patients with acute moderate to severe cholecystitis who are at high risk for preoperative risk, PTGBD should be performed first to alleviate local inflammation and improve systemic function, while cholecystectomy should be delayed. In our clinical practice, this treatment method has achieved good therapeutic effects, not only reducing surgical complications but also reducing mortality.

However, there is still no consensus on when to perform subsequent cholecystectomy after PTGBD and after improvements in systemic function and local inflammation have been achieved. Chikamori et al. (17) found that early LC after PTGBD is safe and effective in patients with acute moderate to severe cholecystitis, especially in elderly and patients with poor basic condition, suggesting the decision should be made on clinical grounds, and long-term drainage is not supported.

Our results showed there were no significant differences in the sex ratio, complication rate, or rate of conversion to laparotomy between the early LC group (group A) and other groups. However, there were differences in age between group A patients and group F, with those in group A being younger. The operation time of the early LC patients group was not prolonged, and the length of hospital stay and hospitalization costs were reduced compared with other groups, showing the benefits to patients in that group were greater than in others. On this basis, we conclude that after a definitive diagnosis is formed, and factors such as contraindications and the clinical state of the patient are 
taken into account, early LC should be performed after clinical symptoms are alleviated.

In circumstances where local fibrotic adhesions in the triangle of gallbladder are formed within 2-8 weeks after PTGBD and edema does not completely disappear, LC should be delayed until clinically appropriate. Our results show that outcomes where PTGBD was delayed for 2 months were not statistically different from when the delay stretched to 3 months. However, extending the time in which a drainage tube is inserted is not recommended.

Studies in recent years have suggested that LC should not be performed in the early stage after PTGBD (18). Many clinical studies have observed the time from LC to 2, 4, 8, and even more than 12 weeks after PTGBD (19). Kim (20) found that in patients with acute cholecystitis who underwent LC 2 weeks after PTGBD, the incidence of conversion to laparotomy and complications was significantly lower than that when LC 1 week after PTGBD was performed. Watanabe et al. (21) reported no conversion to laparotomy by LC 34 days after PTGBD, while Kuster et al. (22) found LC without conversion to laparotomy could be performed 3 months after PTGBD. While this suggests that the longer the time after PTGBD, the lower the conversion to laparotomy rate, with the extension of time, problems such as the length of hospitalization, cost of treatment, and the influence of a drainage tube on normal life become increasingly prominent. In this study, although systemic and local inflammation in patients who received PTGBD for 2-8 weeks was significantly controlled and patients resumed a normal diet and life, the triangle of the gallbladder and hepatoduodenal ligament formed fibrous adhesions, the gallbladder texture became hard, and the operation was more difficult and its time prolonged. Therefore, we should be alert to the anatomical abnormalities caused by severe intraoperative adhesion to prevent bile duct injury.

The results of this study also suggested that if LC was performed more than 2 months after PTGBD, there were no significant differences in gender, age, operation time, length of hospital stay, hospitalization expenses, complication rate and conversion rate to laparotomy compared to patients in whom LC was performed more than 3 months after PTGBD. Therefore, it is not necessary to prolong the time for patients to maintain drainage tubes. When systemic and local conditions improve, the inflammation of the gallbladder triangle will subside over 2 months, and patients should be operated on as soon as possible to reduce the inconvenience of tubes and relieve pain.
There were also some limitations in this study as follows: (I) as the study isretrospective and from a singlecenter, patient selection may not be representative, and the sample size is small. (II) As patients with gallbladder disease admitted to Tianjin Nankai Hospital are difficult to treat or are untreatable in primary hospitals, the final cases may not be typical and lack validation cohort validation.

\section{Conclusions}

In conclusion, for patients diagnosed with acute moderate to severe cholecystitis who meet the indications, LC is recommended to be performed within 1 week following PTGBD surgery. When delayed LC is performed within 2 to 8 weeks after PTGBD, the operation time will be longer due to inflammatory edema and fibrous adhesions of the gallbladder triangle. If patients on whom PTGBD is performed longer than 2 months are in reasonable health, delayed LC can be considered to reduce the inconvenience of long-term catheter.

\section{Acknowledgments}

Funding: This work has been supported partially by the Research Projects in Key Areas of Traditional Chinese Medicine of Tianjin Health Commission (No. 2019003).

\section{Footnote}

Reporting Checklist: The authors have completed the STROBE reporting checklist. Available at https://dx.doi. org/10.21037/apm-21-1906

Data Sharing Statement: Available at https://dx.doi. org/10.21037/apm-21-1906

Conflicts of Interest: All authors have completed the ICMJE uniform disclosure form (available at https://dx.doi. org/10.21037/apm-21-1906). The authors have no conflicts of interest to declare.

Ethical Statement: The authors are accountable for all aspects of the work in ensuring that questions related to the accuracy or integrity of any part of the work are appropriately investigated and resolved. This study was reviewed and approved by the Ethics Committee of Tianjin Nankai Hospital (No. NKYY_YXKT_IRB_2019_004_01). Written informed consent was obtained from all study participants. 
All procedures performed in this study involving human participants were in accordance with the Declaration of Helsinki (as revised in 2013)

Open Access Statement: This is an Open Access article distributed in accordance with the Creative Commons Attribution-NonCommercial-NoDerivs 4.0 International License (CC BY-NC-ND 4.0), which permits the noncommercial replication and distribution of the article with the strict proviso that no changes or edits are made and the original work is properly cited (including links to both the formal publication through the relevant DOI and the license). See: https://creativecommons.org/licenses/by-nc-nd/4.0/.

\section{References}

1. 1. Yokoe M, Hata J, Takada T, et al. Tokyo Guidelines 2018: diagnostic criteria and severity grading of acute cholecystitis (with videos). J Hepatobiliary Pancreat Sci 2018;25:41-54.

2. Okamoto K, Suzuki K, Takada T, et al. Tokyo Guidelines 2018: flowchart for the management of acute cholecystitis. J Hepatobiliary Pancreat Sci 2018;25:55-72.

3. Mori Y, Itoi T, Baron TH, et al. Tokyo Guidelines 2018: management strategies for gallbladder drainage in patients with acute cholecystitis (with videos). J Hepatobiliary Pancreat Sci 2018;25:87-95.

4. Al-Mulhim AA. Timing of early laparoscopic cholecystectomy for acute cholecystitis. JSLS 2008;12:282-7.

5. Sakamoto T, Fujiogi M, Matsui H, et al. Timing of cholecystectomy after percutaneous transhepatic gallbladder drainage for acute cholecystitis: a nationwide inpatient database study. HPB (Oxford) 2020; 22:920-6.

6. Sato N, Kinoshita A, Imai N, et al. Inflammation-based prognostic scores predict disease severity in patients with acute cholecystitis. Eur J Gastroenterol Hepatol 2018;30:484-9.

7. Lee R, Ha H, Han YS, et al. Percutaneous transhepatic gallbladder drainage followed by elective laparoscopic cholecystectomy for patients with moderate to severe acute cholecystitis. Medicine (Baltimore) 2017;96:e8533.

8. Yu B, Zhi X, Li Q, et al. The efficacy and safety of preoperative cholangiography via percutaneous transhepatic gallbladder drainage (PTGBD) for difficult laparoscopic cholecystectomy (LC). Surg Endosc 2021. [Epub ahead of print]. doi: 10.1007/ s00464-021-08414-0.
9. Takada T. Tokyo Guidelines 2018: updated Tokyo Guidelines for the management of acute cholangitis/acute cholecystitis. J Hepatobiliary Pancreat Sci 2018;25:1-2.

10. Kuroki T, Kitasato A, Tokunaga T, et al. Results of elective laparoscopic cholecystectomy for acute cholecystitis following percutaneous transhepatic gallbladder drainage. Acta Medica Nagasakiensia 2018;61:111-5.

11. Cullen JJ, Scott-Conner CEH. Surgical anatomy of laparoscopic common duct exploration. In: Berci G, Cuschieri A. editors. Bile ducts and bile duct stones. Philadelphia: WB Saunders, 1997:20-5.

12. Farooq T, Buchanan G, Manda V, et al. Is early laparoscopic cholecystectomy safe after the "safe period"? J Laparoendosc Adv Surg Tech A 2009;19:471-4.

13. Rogers PN. Long-term outcome of patients with acute cholecystitis receiving antibiotic treatment: a retrospective cohort study. World J Surg 2014;38:355-6.

14. Radder RW. Ultrasonically guided percutaneous catheter drainage for gallbladder empyema. Diagn Imaging 1980;49:330-3.

15. Melloul E, Denys A, Demartines N, et al. Percutaneous drainage versus emergency cholecystectomy for the treatment of acute cholecystitis in critically ill patients: does it matter? World J Surg 2011;35:826-33.

16. Hung YL, Chong SW, Cheng CT, et al. Natural course of acute cholecystitis in patients treated with percutaneous transhepatic gallbladder drainage without elective cholecystectomy. J Gastrointest Surg 2020;24:772-9.

17. Chikamori F, Kuniyoshi N, Shibuya S, et al. Early scheduled laparoscopic cholecystectomy following percutaneous transhepatic gallbladder drainage for patients with acute cholecystitis. Surg Endosc 2002;16:1704-7.

18. Zhao H, Liu K, Liu Y. The most appropriate timing for selective laparoscopic cholecystectomy after percutaneous transhepatic gallbladder drainage in patients with acute severe cholecystitis aged above 60 years. Journal of Clinical Hepatology 2017;33:705-10.

19. El-Gendi A, El-Shafei M, Emara D. Emergency versus delayed cholecystectomy after percutaneous transhepatic gallbladder drainage in grade II acute cholecystitis patients. J Gastrointest Surg 2017;21:284-93..

20. Kim HO, Ho Son B, Yoo CH, et al. Impact of delayed laparoscopic cholecystectomy after percutaneous transhepatic gallbladder drainage for patients with complicated acute cholecystitis. Surg Laparosc Endosc Percutan Tech 2009;19:20-4.

21. Watanabe Y, Sato M, Abe Y, et al. Preceding PTGBD decreases complications of laparoscopic cholecystectomy 
for patients with acute suppurative cholecystitis. J

Laparoendosc Surg 1996;6:161-5.

22. Kuster GG, Domagk D. Laparoscopic cholecystostomy with delayed cholecystectomy as an alternative to conversion to open procedure. Surg Endosc 1996;10:426-8.

(English Language Editor: B. Draper)

Cite this article as: Bao J, Wang J, Shang H, Hao C, Liu J, Zhang D, Han S, Li Z. The choice of operation timing of laparoscopic cholecystectomy (LC) after percutaneous transhepatic gallbladder drainage (PTGBD) for acute cholecystitis: a retrospective clinical analysis. Ann Palliat Med 2021;10(8):9096-9104. doi: 10.21037/apm-21-1906 